\title{
On coloring problems with local constraints
}

\author{
Flavia Bonomo ${ }^{\mathrm{a}, \mathrm{b}, 1}$, Yuri Faenza ${ }^{\mathrm{a}, 2}$, Gianpaolo Oriolo ${ }^{\mathrm{a}, 2}$ \\ ${ }^{a}$ Università di Roma "Tor Vergata", Dipartimento di Ingegneria dell'Impresa, via del \\ Politecnico 1, 00133, Roma, Italy \\ ${ }^{b}$ CONICET and Departamento de Computación, FCEyN, Universidad de Buenos Aires, \\ Buenos Aires, Argentina
}

\begin{abstract}
We deal with some generalizations of the graph coloring problem on classes of perfect graphs. Namely we consider the $\mu$-coloring problem (upper bounds for the color on each vertex), the precoloring extension problem (a subset of vertices colored beforehand), and a problem generalizing both of them, the $(\gamma, \mu)$-coloring problem (lower and upper bounds for the color on each vertex). We characterize the complexity of all those problems on clique trees of different heights, providing polytime algorithms for the cases that are easy. These results have two interesting corollaries: first, one can observe on clique trees of different heights the increasing complexity of the chain $k$-coloring, $\mu$-coloring, $(\gamma, \mu)$-coloring, list-coloring. Second, clique trees of height 2 are the first known example of a class of graphs where $\mu$-coloring is polynomial time solvable and precoloring extension is NP-complete, thus being at the same time the first example where $\mu$-coloring is polynomially solvable and $(\gamma, \mu)$-coloring is NP-complete. Last, we show that the $\mu$-coloring problem on unit interval graphs is NP-complete. These results answer three questions from [Ann. Oper. Res. 169(1) (2009), 3-16].
\end{abstract}

Key words: Graph coloring, clique trees, unit interval graphs, computational complexity.

Email addresses: fbonomo@dc.uba.ar (Flavia Bonomo), faenza@disp.uniroma2.it (Yuri Faenza), oriolo@disp.uniroma2.it (Gianpaolo Oriolo)

${ }^{1}$ Partially supported by ANPCyT PICT-2007-00518 and PICT-2007-00533, and UBACyT Grants X069 and X606 (Argentina).

${ }^{2}$ Partially supported by ANPCyT PICT-2007-00533. 


\section{Introduction}

A coloring of a graph $G(V, E)$ is a function $f: V \rightarrow \mathbb{N}$ such that $f(v) \neq$ $f(w)$ whenever $v w \in E$. A $k$-coloring is a coloring $f$ such that $f(v) \leq k$ for every $v \in V$. The vertex coloring problem takes as input a graph $G$ and a natural number $k$, and consists in deciding whether $G$ is $k$-colorable or not. This well-known problem is a basic model for scheduling, frequency assignment and resource allocation problems.

In order to take into account particular constraints arising in practical settings, more elaborate models of vertex coloring have been defined in the literature. A hierarchy of such models was studied in [4]. Two generalizations of the $k$-coloring problem are precoloring extension [2] and $\mu$ coloring [3].

The precoloring extension problem takes as input a graph $G(V, E)$, a subset $W \subseteq V$, a natural number $k$, a $k$-coloring $f^{\prime}$ of $W$, and consists in deciding whether $G$ admits a $k$-coloring $f$ such that $f(v)=f^{\prime}(v)$ for every $v \in W$ or not. In other words, a prespecified vertex subset is colored beforehand, and the goal is to extend this partial coloring to a valid $k$ coloring of the whole graph.

Given a graph $G(V, E)$ and a function $\mu: V \rightarrow \mathbb{N}$, the $\mu$-coloring problem consists in deciding whether $G$ is $\mu$-colorable, i.e. whether there exists a function $f: V \rightarrow \mathbb{N}$ such that $f(v) \leq \mu(v)$ for every $v \in V$ and $f$ is a $k$-coloring of $G$ for some natural $k$. This model arises in resources allocation problems with conflict between users [3], as well as in railways train scheduling [5].

A problem generalizing the latter two ones is the $(\gamma, \mu)$-coloring problem [4], where also lower bounds for the color of each vertex are determined: given a graph $G(V, E)$ and functions $\gamma, \mu: V \rightarrow \mathbb{N}$ such that $\gamma(v) \leq \mu(v)$ for every $v \in V$, the $(\gamma, \mu)$-coloring problem consists in deciding whether there exists a $\mu$-coloring $f$ where additionally $\gamma(v) \leq f(v)$ for every $v \in V$.

Finally, a model generalizing all of the previous problems is the listcoloring problem [20], which considers a prespecified set of available colors for each vertex. Given a graph $G$ and a finite list $L(v) \subseteq \mathbb{N}$ for each vertex $v \in V$, the list-coloring problem asks for a list-coloring of $G$, i.e., a coloring $f$ such that $f(v) \in L(v)$ for every $v \in V$.

The scheme of generalizations, summarized in Figure 1, implies that all the problems in this hierarchy are polynomially solvable in those graph classes where list-coloring is polynomial and, on the other hand, all the problems are NP-complete in those graph classes where vertex coloring is NP-complete. 


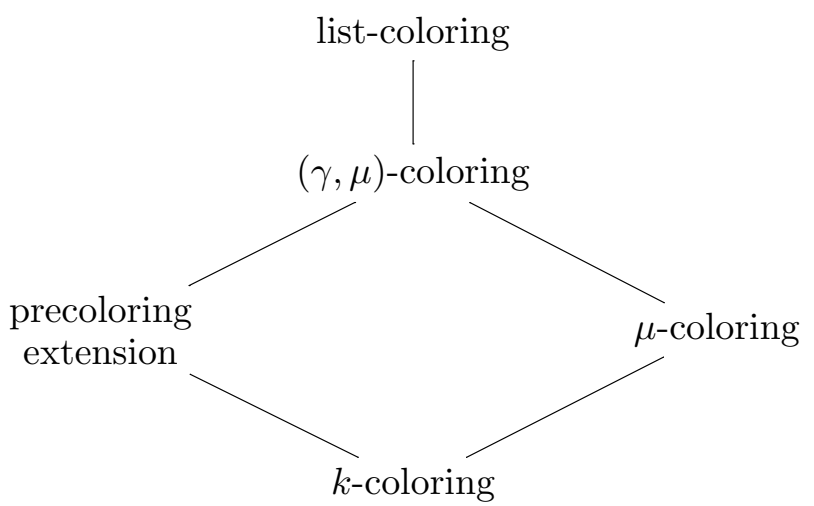

Figure 1: Scheme of generalizations among these coloring problems.

The complexity of this family of problems over different classes of graphs has been studied, and there are several examples of classes where $k$-coloring is polynomial-time solvable but precoloring extension and $\mu$-coloring are NPcomplete, like bipartite graphs [3, 10], interval graphs $[2,4,6]$ and distance hereditary graphs [4, 9], where precoloring extension is polynomial-time solvable but $(\gamma, \mu)$-coloring is NP-complete, like split graphs [11,4], and where $(\gamma, \mu)$-coloring is polynomial time solvable but list-coloring is NP-complete, like complete bipartite graphs and complete split graphs [4, 13]. Note that, however, to the best of our knowledge, so far no class of graphs where $(\gamma, \mu)$-coloring is NP-complete while $\mu$-coloring is polynomially solvable was known, and finding it is mentioned as an open problem in [4].

The problems of precoloring extension and $\mu$-coloring are not directly related, i.e., no one is a generalization of the other one. Nevertheless, for almost all the graph classes where their complexity is known, they are in the same side of the dichotomy "polynomial time solvable vs. NP-complete". The class of split graphs is the only known exception where precoloring extension is polynomial time solvable [11] while $\mu$-coloring is NP-complete [4]. Again, to the best of our knowledge, so far no class of graphs where $\mu$ coloring is polynomially solvable and precoloring extension is NP-complete was known and finding it is mentioned as another open problem in [4].

In this work we study the complexity of these coloring problems on a class of perfect graphs called clique trees. We characterize the complexity of each problem on clique trees of different heights, providing polytime algorithms for the cases that are easy. These results have two interesting corollaries: first, one can observe on clique trees of different heights the increasing 
complexity of the chain $k$-coloring, $\mu$-coloring, $(\gamma, \mu)$-coloring, list-coloring. Second, clique trees of height 2 are the first known example of a class of graphs where $\mu$-coloring is polynomial time solvable and precoloring extension is NP-complete, thus being at the same time the first example where $\mu$-coloring is polynomially solvable and $(\gamma, \mu)$-coloring is NP-complete; this solves the above questions from [4].

We also study the complexity of the $\mu$-coloring problem on another class of perfect graphs, the one of unit interval graphs. Both the precoloring extension problem and the $\mu$-coloring problem were motivated by and arise in the context of scheduling problems, like job scheduling [2], classroom allocation [3] and railways train scheduling [5]. Thus one of the classes of interest for these problems is the class of interval graphs and, in particular, the class of unit interval graphs.

It is well known that the chromatic number of an interval graph $G(V, E)$ can be determined in time $O(|V|+|E|)$. While the NP-completeness of precoloring extension on interval graphs was proved in 1992 [2], it took more than ten years, and a quite involved reduction, in order to prove that the problem is hard also for unit interval graphs [15]. Analogously, in [4] it was proved that the $\mu$-coloring problem is NP-complete on interval graphs and the question of the complexity of $\mu$-coloring on unit interval graphs was raised. We also settle this question and show, via a non-trivial reduction, that this problem is NP-complete.

The paper is organized as follows: we close this section with some notation. In Section 2 we introduce the class of clique trees and the related notion of height, characterizing the complexity of the aforementioned coloring problems on clique trees of different heights. In Section 3, we present a (quite involved) proof of the NP-completeness of the $\mu$-coloring problem on unit interval graphs.

We shall consider finite, simple, loopless, undirected graphs. Let $G$ be a graph. Denote by $V(G)$ its vertex set and by $E(G)$ its edge set. Given a vertex $v$ of $G$, denote by $N_{G}(v)$ the set of neighbors of $v$ in $G$ and by $N_{G}[v]$ the set $N_{G}(v) \cup\{v\}$, and generalize it to a set of vertices $W \subseteq V$ as follows: $N_{G}(W)=\cup_{w \in W} N_{G}(w)$, and $N_{G}[W]=N_{G}(W) \cup W$. A graph $G$ is a tree if it is connected and $|E(G)|=|V(G)|-1$ holds. A rooted tree is a pair $(G, r)$ consisting of a tree $G$ together with a designated vertex $r \in V(G)$. Two vertices $v$ and $w$ of $G$ are said to be true twins if either $v=w$ or $N_{G}[v]=N_{G}[w]$, i.e., they are adjacent and they have the same neighbors.

For any $W \subseteq V(G)$, denote by $G[W]$ the subgraph of $G$ induced by $W$. If 
$H$ is an induced subgraph of $G$, denote by $G \backslash H$ the graph $G[V(G) \backslash V(H)]$.

Given two graphs $G_{1}, G_{2}$ with $V\left(G_{1}\right) \cap V\left(G_{2}\right)=\emptyset$, we will denote by $G_{1} \cup G_{2}$ a graph such that $V\left(G_{1} \cup G_{2}\right)=V\left(G_{1}\right) \cup V\left(G_{2}\right)$ and $E\left(G_{1} \cup G_{2}\right)=$ $E\left(G_{1}\right) \cup E\left(G_{2}\right)$. Given a graph $G$ and two vertices $v, w$ of it, contracting them into a single vertex will mean to replace them by a new vertex, and making it adjacent to the neighbors of $v$ and $w$ in $G \backslash\{v, w\}$.

For a strictly positive integer $k$, let $[k]=\{1,2, \ldots, k\}$.

Given two vertices $a, b \in V(G)$, an $(a, b)$-path of length $k$ in $G$ is an ordered sequence of vertices $\left\{v_{0}, v_{1}, \ldots, v_{k}\right\}$ such that $v_{0}=a, v_{k}=b$, all vertices are distinct, and for $i \in[k],\left(v_{i-1}, v_{i}\right) \in E(G)$. The distance of $(a, b)$ in $G$ is the minimum length of all $(a, b)$-paths in $G$.

A clique is a set of pairwise adjacent vertices. Denote by $K_{n}$ the clique of size $n$. A stable set is a set of pairwise non-adjacent vertices. Let $A, B \subseteq$ $V(G)$. We say that $A$ is complete to $B$ if every vertex of $A$ is adjacent to every vertex of $B$; and $A$ is anticomplete to $B$ if no vertex of $A$ is adjacent to a vertex of $B$.

The chromatic number of a graph $G$ is the minimum $k$ such that $G$ is $k$-colorable. A graph is perfect [1] if for every induced subgraph $H$ of it, the chromatic number of $H$ coincides with the size of a maximum clique of $H$. The $k$-coloring problem is known to be polynomial time solvable on perfect graphs [7].

Throughout the paper, an instance of the $\mu$-coloring will be a pair $(G, \mu)$, where $\mu: V(G) \rightarrow \mathbb{N}$; an instance of the $(\gamma, \mu)$-coloring will be a triple $(G, \gamma, \mu)$, where $\mu, \gamma: V(G) \rightarrow \mathbb{N}$ with $\gamma(v) \leq \mu(v)$ for each $v \in V(G)$; an instance of the precoloring extension will be a 4 -tuple $\left(G, W, f^{\prime}, k\right)$ where $W \subseteq V(G), k \in \mathbb{N}$ and $f^{\prime}: W \rightarrow[k]$ is a valid coloring of $G[W]$; an instance of list-coloring will be a pair $(G, L)$, where $L: V(G) \rightarrow 2^{(\mathbb{N})}$, i.e., $L(v)$ is finite for each $v \in V(G)$.

\section{Clique trees}

A graph $G$ is a clique tree if the graph $G^{\prime}$ obtained by iteratively contracting its true twins is a tree. It is immediate to check that $G^{\prime}$ does not depend on the order of the contractions, and thus it is well defined. The height of a rooted tree is the maximum distance from the root to any other vertex of the tree. The height of a tree is the minimum height over all the choices of a root. The height of a clique tree $G$ is the height of the tree obtained by identifying true twins in $G$. We shall denote that tree by $\mathcal{T}(G)$, and call root any vertex of $\mathcal{T}(G)$ for which the minimum height is achieved. 
Given a vertex $v$ of $G$, the multiplicity of $v$ in $G$ is the number of true twins of $v$ in $G$ (by definition, $v$ is also counted).

Clique trees are perfect graphs since trees are perfect graphs and the operation of adding true twins maintains perfection [14]. Moreover, the size of the maximum clique, an thus the chromatic number, is the maximum sum of the multiplicities of two adjacent vertices that are not twins. So the $k$-coloring problem can be solved in strongly polynomial time for this class of graphs.

As for the other coloring problems we are interested in this paper, the complexity changes with the height of the clique tree. Clique trees of height 0 are the complete graphs, and the list-coloring problem on a complete graph can be modelled as a maximum matching problem on a bipartite graph and thus solved in polynomial time.

Meanwhile, it is known that the list-coloring problem is NP-complete for clique trees of height 1 , even if its corresponding tree is formed by a root and two children [12], or if the multiplicity of all the vertices but the root is 1 [13]. Vice versa, as we show in the following, $(\gamma, \mu)$-coloring, and therefore $\mu$-coloring and precoloring extension, are still easy for clique trees of height 1.

If we move to clique trees of height 2 , we show in the following that only $\mu$-coloring (and, of course, $k$-coloring) is easy. As mentioned in the introduction, this class of graphs gives, to the best of our knowledge, the first known example where $\mu$-coloring is polynomially solvable, while precoloring extension (and thus $(\gamma, \mu)$-coloring) is NP-complete.

If we then move to height 3 or more, then also $\mu$-coloring becomes hard, even when the height is fixed. Table 2 summarizes these results. Interestingly, one can observe on clique trees of different heights the increasing complexity of the chain $k$-coloring, $\mu$-coloring, $(\gamma, \mu)$-coloring, list-coloring.

\begin{tabular}{l|llll} 
Problem & \multicolumn{4}{|c}{ Height } \\
& 0 & 1 & 2 & fixed $p \geq 3$ \\
\hline$k$-coloring & $\mathrm{P}$ & $\mathrm{P}$ & $\mathrm{P}$ & $\mathrm{P}$ \\
$\mu$-coloring & $\mathrm{P}$ & $\mathbf{P}$ & $\mathbf{P}$ & NP-c \\
precoloring & $\mathrm{P}$ & $\mathbf{P}$ & $\mathbf{N P}-\mathbf{c}$ & NP-c \\
$(\gamma, \mu)$-coloring & $\mathrm{P}$ & $\mathbf{P}$ & NP-c & NP-c \\
list-coloring & $\mathrm{P}$ & NP-c & NP-c & NP-c \\
\hline
\end{tabular}

Table 1: A summary of the complexity results for clique trees. Original results from this paper are bold-faced.

We start with some useful lemmas. 
Lemma 1. For a strictly positive integer n, let $G$ be a complete graph with vertex set $V \cup V^{\prime}$, where $V=\left\{v_{1}, \ldots, v_{n}\right\}$ and $V^{\prime}=\left\{v_{1}^{\prime}, \ldots, v_{n}^{\prime}\right\}$. Let $\mu: V(G) \rightarrow \mathbb{N}$ such that $\mu\left(v_{i}\right)=\mu\left(v_{i}^{\prime}\right)=2 i$ for $i$ in $[n]$. Let $\gamma: V \rightarrow \mathbb{N}$ such that $\gamma\left(v_{i}\right)=2 i-1$ for $i$ in $[n]$. If $f$ is a solution to $(G, \mu)$, then its restriction to $G[V]$ is a solution to $(G[V],(\gamma, \mu))$. Vice versa, any solution to $(G[V],(\gamma, \mu))$ can be extended in polytime to a solution of $(G, \mu)$.

Proof. Consider a feasible solution to $(G, \mu)$ and let $i \in[n]$. Since $G$ is complete and, for every $j \in[n], \mu\left(v_{j}\right)=\mu\left(v_{j}^{\prime}\right)=2 j$, it follows that vertices $v_{1}, \ldots, v_{i}, v_{1}^{\prime}, \ldots, v_{i}^{\prime}$ use all the colors in [2i]. Thus vertex $v_{i+1}$ uses either color $2 i+1$ or $2(i+1)$. Then the restriction to $V$ gives a solution to $(G[V],(\gamma, \mu))$. Conversely, a solution to $(G[V],(\gamma, \mu))$ leaves unused either $2 i$ or $2 i-1$ for each $i \in[n]$, so we can extend it to a solution of $(G, \mu)$ by assigning that free color to $v_{i}^{\prime}$.

The following Lemma is implicitly proved in [4], see Theorem 4.

Lemma 2. Let $G$ be a complete graph and let $\gamma, \mu: V(G) \rightarrow \mathbb{N}$ such that for every $w \in V(G), \gamma(w) \leq \mu(w)$. Let $\mu_{\max }=\max _{w \in V(G)} \mu(w)$. Let $C$ be a subset of natural numbers within the interval $\left[1, \mu_{\max }\right]$. Then $G$ admits a $(\gamma, \mu)$-coloring using only colors in $C$ if and only if, for every $1 \leq i \leq j \leq \mu_{\max },|\{w \in V(G): i \leq \gamma(w) \leq \mu(w) \leq j\}| \leq|C \cap[i, j]|$

\subsection{Polynomial cases}

Theorem 3. The $(\gamma, \mu)$-coloring problem can be solved in polynomial time for clique trees of height at most 1.

Proof. The statement is true for clique trees of height 0 , i.e. complete graphs, since there the more general list-coloring problem can be modelled as a maximum matching problem on a bipartite graph.

Suppose therefore that we are given a graph $G$ that is a clique tree of height 1 and $\gamma, \mu: V(G) \rightarrow \mathbb{N}$ such that for every $v \in V(G), \gamma(v) \leq \mu(v)$. Let $\mu_{\max }$ be the maximum value of $\mu$ over $G$. We may assume that every color between 1 and $\mu_{\max }$ belongs to the interval $[\gamma(v), \mu(v)]$, for some $v \in V(G)$. Furthermore, we may assume $\mu(v)-\gamma(v) \leq\left|N_{G}(v)\right|$ for every $v \in V(G)$. It follows from these assumptions that $\mu_{\max } \leq n \cdot \max _{v \in V}\left|N_{G}(v)\right|$.

Let $A$ be the clique corresponding to the root of $\mathcal{T}(G)$ and $B_{1}, \ldots, B_{r}$ the cliques corresponding to the leaves of $\mathcal{T}(G)$. For $0<j \leq i \leq \mu_{\max }$, let $L_{A}(i, j)=|\{v \in A: j \leq \gamma(v) \leq \mu(v) \leq i\}|$ and $L_{k}(i, j)=\mid\left\{v \in B_{k}\right.$ : $j \leq \gamma(v) \leq \mu(v) \leq i\} \mid$, for $k$ in $[r]$. We reduce the problem of finding a $(\gamma, \mu)$-coloring of $G$ to a linear programming feasibility problem. For $j$ in 
[ $\left.\mu_{\max }\right]$, we define the integer variable $x_{j}$ to be the number of colors from the set $[j]$ assigned to vertices of $A$ and, based on this definition, we consider the following linear program:

$$
\begin{aligned}
x_{0} & =0 & & \\
x_{i}-x_{j-1} & \geq L_{A}(i, j) & & \forall i, j: 0<j \leq i \leq \mu_{\max } \\
x_{i}-x_{j-1} & \leq i-j+1-\max _{k \in[r]} L_{k}(i, j) & & \forall i, j: 0<j \leq i \leq \mu_{\max }
\end{aligned}
$$

Since $\mu_{\max } \leq n \cdot \max _{v \in V}\left|N_{G}(v)\right|$, it follows that the number of variables and constraints is polynomial in the size of $G$. All the constraints take the form $x_{j}-x_{k} \geq \alpha_{j k}$ or $x_{j}=\alpha_{j}$, hence the constraint matrix is totally unimodular, implying that the associated polytope is integral (see for example [16]). To complete the proof, we verify that $G$ is $(\gamma, \mu)$-colorable if and only if the linear program (1)-(3) is feasible.

Assume first $G$ is $(\gamma, \mu)$-colorable. Let $x_{0}=0$ and, for $j$ in $\left[\mu_{\max }\right]$, let $x_{j}$ be the number of colors from $[j]$ assigned to vertices of $A$. Constraint (1) holds trivially. Since $A$ is a clique, at least $L_{A}(i, j)$ colors from $[j, i]$ are assigned to the vertices of $A$, hence constraints (2) hold. Analogously, for each $k \in[r]$, at least $L_{k}(i, j)$ colors from $[j, i]$ are assigned to the vertices of $B_{k}$; then, since each $B_{k}$ is complete to $A$, constraints (3) hold. Thus, if $G$ is $(\gamma, \mu)$-colorable, then the linear program (1)-(3) has a feasible solution.

Conversely, assume that the linear program (1)-(3) is feasible and let $x$ be an integer solution, which exists since the associated polytope is integral. We shall verify that $G$ admits a $(\gamma, \mu)$-coloring. Let $C=\left\{j: 1 \leq j \leq \mu_{\max }\right.$ and $\left.x_{j}-x_{j-1}=1\right\}$. By (2), $C$ and $A$ satisfy the hypothesis of Lemma 2 , so there is a $(\gamma, \mu)$-coloring of $A$ using the colors in $C$. By (3), for each $k$ in $[r]$, the complete graph $B_{k}$ and the set of colors $\left[\mu_{\max }\right] \backslash C$ satisfy the hypothesis of Lemma 2 , so there is a $(\gamma, \mu)$-coloring of $B_{k}$ using the colors in $\left[\mu_{\max }\right] \backslash C$. Putting things together, there exists a $(\gamma, \mu)$-coloring of $G$; note that this coloring may be found solving $r+1$ bipartite matching problems.

Since the $(\gamma, \mu)$-coloring problem is a generalization of the precoloring extension and the $\mu$-coloring problem, we have the following corollary.

Corollary 4. The precoloring extension and the $\mu$-coloring problem can be solved in polynomial time for clique trees of height at most 1.

Theorem 5. The $\mu$-coloring problem can be solved in polynomial time for clique trees of height at most 2. 
Proof. Let $(G, \mu)$ be an instance of $\mu$-coloring where $G$ is a clique tree of height 2. We shall show that $(G, \mu)$ can be polynomially reduced to an instance $\left(G^{\prime},(\gamma, \mu)\right)$ of the $(\gamma, \mu)$-coloring problem, where $G^{\prime}$ is a clique tree of height 1 . Then we can invoke Theorem 3 .

Let $A$ be the clique of $G$ corresponding to the root of $\mathcal{T}(G),\left\{B_{k}\right\}_{k \in[r]}$ the cliques of $G$ corresponding to the vertices in level 1 of $\mathcal{T}(G),\left\{C_{k}^{j}\right\}_{k \in[r], j \in\left[s_{k}\right]}$ the cliques of $G$ corresponding to the leaves of $\mathcal{T}(G)$, where for each $k$ in $[r]$ and each $j$ in $\left[s_{k}\right], C_{k}^{j}$ is complete to $B_{k}$. The graph $G^{\prime}$ is obtained from $G$ by deleting the cliques corresponding to vertices of level 2 in $\mathcal{T}(G)$, i.e. $G^{\prime}=G\left[A \cup_{k \in[r]} B_{k}\right]$. Thus $G^{\prime}$ is a clique tree of height 1 .

As for the vector $\gamma$, it requires some more definitions. Let $\mu_{\max }$ be the maximum value of $\mu$ over $G$ and, for every $i \in\left[\mu_{\max }\right]$ and $k \in[r]$, let $l_{i}^{k}=$ $\max _{1 \leq j \leq s_{k}}\left|\left\{v \in C_{k}^{j}: \mu(v) \leq i\right\}\right|$. Also we assume that $B_{k}=\left\{w_{k}^{1}, \ldots, w_{k}^{\left|B_{k}\right|}\right\}$, with $\mu\left(w_{k}^{1}\right) \leq \cdots \leq \mu\left(w_{k}^{\left|B_{k}\right|}\right)$, for $k \in[r]$. For each $k \in[r]$ and $j \in\left[\left|B_{k}\right|\right]$, we let $\gamma\left(w_{k}^{j}\right)=h$, where $(h-1)$ is the largest index $i \in\left[\mu_{\max }\right]: l_{k}^{i}+j>i$. Meanwhile, we let $\gamma(v)=1$ for every $v \in A$.

We claim that the resulting $\gamma$-vector is such that, for $i \in\left[\mu_{\max }\right], \mid\{v \in$ $\left.B_{k}: \gamma(v) \leq i\right\} \mid \leq i-l_{k}^{i}$. Indeed, fix $i$ and suppose $i-l_{k}^{i}<\left|B_{k}\right|$. Then for $w=w_{k}^{i-l_{i}^{k}+1}, \ldots, w_{k}^{\left|B_{k}\right|}, \gamma(w) \geq i+1$, so the statement holds. Conversely if $i-l_{k}^{i} \geq\left|B_{k}\right|$, then the statement holds since $\left|\left\{v \in B_{k}: \gamma(v) \leq i\right\}\right|$ is a subset of $B_{k}$.

We finally show that every solution of $\left(G^{\prime},(\gamma, \mu)\right)$ can be extended to a solution of $(G, \mu)$ and, conversely, every solution to $(G, \mu)$ is, restricted to $V\left(G^{\prime}\right)$, a solution of $\left(G^{\prime},(\gamma, \mu)\right)$. Let $f$ be a $(\gamma, \mu)$-coloring of $G^{\prime}$. It is, in particular, a $\mu$-coloring of $G^{\prime}$. For each $k \in[r]$, let $f\left(B_{k}\right)=\left\{f(v), v \in B_{k}\right\}$ and let $Q_{k}=\left[\mu_{\max }\right] \backslash f\left(B_{k}\right)$. We claim that, for each $k \in[r]$ and $j \in\left[s_{k}\right]$, there exists a $\mu$ coloring of $C_{k}^{j}$ that uses only colors from $Q_{k}$. From Lemma 2 , it is enough to show that, for every $i \leq \mu_{\max },\left|\left\{v \in C_{k}^{j}: \mu(w) \leq i\right\}\right| \leq$ $\left|Q_{k} \cap[i]\right|=i-\left|f\left(B_{k}\right) \cap[i]\right|$. Since $\left|f\left(B_{k}\right) \cap[i]\right| \leq\left|\left\{v \in B_{k}: \gamma(v) \leq i\right\}\right| \leq$ $i-l_{k}^{i} \leq i-\left|\left\{v \in C_{k}^{j}: \mu(v) \leq i\right\}\right|$, we are done.

Conversely, let $f$ be a $\mu$-coloring of $G$. Since, for each $k \in[r], \mu\left(w_{k}^{i}\right) \leq$ $\mu\left(w_{k}^{i+1}\right)$ and vertices in $B_{k}$ are twins, we can permute colors of vertices in $B_{k}$ to obtain $f\left(w_{k}^{1}\right)<\cdots<f\left(w_{k}^{\left|B_{k}\right|}\right)$, without affecting the feasibility of the problem. We claim that $f$ now induces a $(\gamma, \mu)$-coloring of $G^{\prime}$. Note that we only need to show that, for each $k \in[r]$ and $j \in\left[\left|B_{k}\right|\right]$ we have that $\gamma\left(w_{k}^{j}\right) \leq f\left(w_{k}^{j}\right)$. Suppose the contrary and let $h=\gamma\left(w_{k}^{j}\right)>f\left(w_{k}^{j}\right)$ for some $j$. By definition of $\gamma\left(w_{k}^{j}\right)$, it follows that $l_{k}^{h-1}+j>h-1$, i.e. $j>h-1-l_{k}^{h-1}$, and, since $w_{k}^{1}, \ldots w_{k}^{j}$ are receiving a color in $[h-1]$, this is a contradiction 
with $f$ being a feasible $\mu$-coloring of $G$.

\subsection{NP-complete cases}

Theorem 6. For each integer $p \geq 3$, the $\mu$-coloring problem is NP-complete on clique trees of height $p$ and the precoloring extension problem is NPcomplete on clique trees of height $p-1$.

Proof. The proof is based on a reduction from 3-SAT; namely, we are given a 3-SAT instance with variables $x_{1}, \ldots x_{n}$ and clauses $c_{1}, \ldots, c_{k}$. W.l.o.g. we assume that there is no clause $c_{j}$ where both $x_{i}$ and $\bar{x}_{i}$ appear.

Our first task is producing a list-coloring instance $(G, L)$ such that there exists a feasible coloring for $(G, L)$ if and only if there exists a feasible solution to the 3-SAT instance. This goes as follows. Define a bijection $\varphi$ between the set $\cup_{i}\left\{x_{i}, \bar{x}_{i}\right\}$ and $[2 n]$ such that: $\varphi\left(x_{i}\right)=2 i-1, \varphi\left(\bar{x}_{i}\right)=2 i$. The list-coloring instance $(G, L)$ is defined as follows: $V(G)=\{A \cup C\}$, $A=\left\{v_{1}, \ldots, v_{n}\right\}, C=\left\{w_{1}, \ldots, w_{k}\right\}$, where $A$ is a clique, $C$ is a stable set and $A$ is complete to $C$. Let $L\left(v_{i}\right)=\{2 i-1,2 i\}=\left\{\varphi\left(x_{i}\right), \varphi\left(\bar{x}_{i}\right)\right\}$ for $i \in[n]$ and $L\left(w_{j}\right)=\left\{\varphi\left(x_{i}\right) \mid x_{i} \in c_{j}\right\} \cup\left\{\varphi\left(\bar{x}_{i}\right) \mid \bar{x}_{i} \in c_{j}\right\}$ for $j \in[k]$.

Consider now a solution $S$ to the 3-SAT instance: if $x_{i}$ is true (resp. $\bar{x}_{i}$ is true) in $S$, then color $v_{i}$ with $\varphi\left(\bar{x}_{i}\right)$ (resp. $\varphi\left(x_{i}\right)$ ). As for the vertices of $C$, let $c_{j}$ be any clause and suppose $x_{i}$ (resp. $\bar{x}_{i}$ ) makes $c_{j}$ true in $S$; then the color $\varphi\left(x_{i}\right)$ (resp. $\varphi\left(\bar{x}_{i}\right)$ ) is not used in $A$, so it can be used to color $w_{j}$ : thus, we obtain a feasible coloring for $(G, L)$. Conversely, every feasible coloring $f$ to $(G, L)$ induces a true/false assignment to the variables $x_{1}, \ldots x_{n}$ such that the boolean formula is satisfied: namely, make $x_{i}$ true (resp. $\bar{x}_{i}$ true) if and only if the color of $v_{i}$ is $\varphi\left(\bar{x}_{i}\right)$ (resp. $\varphi\left(x_{i}\right)$ ).

We now define an instance $\left(G^{\prime}, \mu\right)$ of the $\mu$-coloring problem such that the list-coloring instance $(G, L)$ has a solution if and only if $\left(G^{\prime}, \mu\right)$ has a solution, where $G^{\prime}$ is a clique tree of height 3 .

$G^{\prime}$ is defined as follows. $V\left(G^{\prime}\right)=A \cup B \cup C \cup D \cup E$, where $A, B, C, D$ and $E$ are pairwise disjoint set of vertices. In particular, $A$ and $C$ are the same sets defined for $G, B=\left\{v_{1}^{\prime}, \ldots, v_{n}^{\prime}\right\}$ is a clique, $D$ and $E$ are both collection of pairwise disjoint set of cliques, that are defined as follows. For each $j$ in $[k]$, let us name the elements in the list $L\left(w_{j}\right)$ as $\ell_{j}^{1}, \ell_{j}^{2}, \ell_{j}^{3}$, where $0=\ell_{j}^{0}<\ell_{j}^{1}<\ell_{j}^{2}<\ell_{j}^{3}$. Then, $D=D_{1} \cup D_{2} \cup \ldots \cup D_{k}$, where, for $j \in[k]$, $D_{j}=D_{j}^{1} \cup D_{j}^{2} \cup D_{j}^{3}$ and each $D_{j}^{i}$ is clique of size $\ell_{j}^{i}-\ell_{j}^{i-1}-1$, for $i \in[3]$. Analogously, $E=E_{1} \cup E_{2} \cup \ldots \cup E_{k}$, where, for $j \in[k], E_{j}=E_{j}^{2} \cup E_{j}^{3}$ and each $E_{j}^{i}$ is a clique of size $\ell_{j}^{i-1}$, for $i \in\{2,3\}$. Note that each set $D_{j}^{i}$ is non-empty, since we are assuming there is no clause $c_{j}$ with both $x_{i}$ and $\bar{x}_{i}$. 
As for $E\left(G^{\prime}\right)$, it is defined as follows. $G^{\prime}[A \cup C]=G, B$ is complete to $A$, for each suitable pair $(i, j), D_{j}^{i}$ is complete to $\left\{w_{j}\right\}$ and $E_{j}^{i}$ is complete to $D_{j}^{i}$, and there are no more edges in $G^{\prime}$ (see Figure 2). Note that $G^{\prime}$ is clique tree of height 3 , as required.

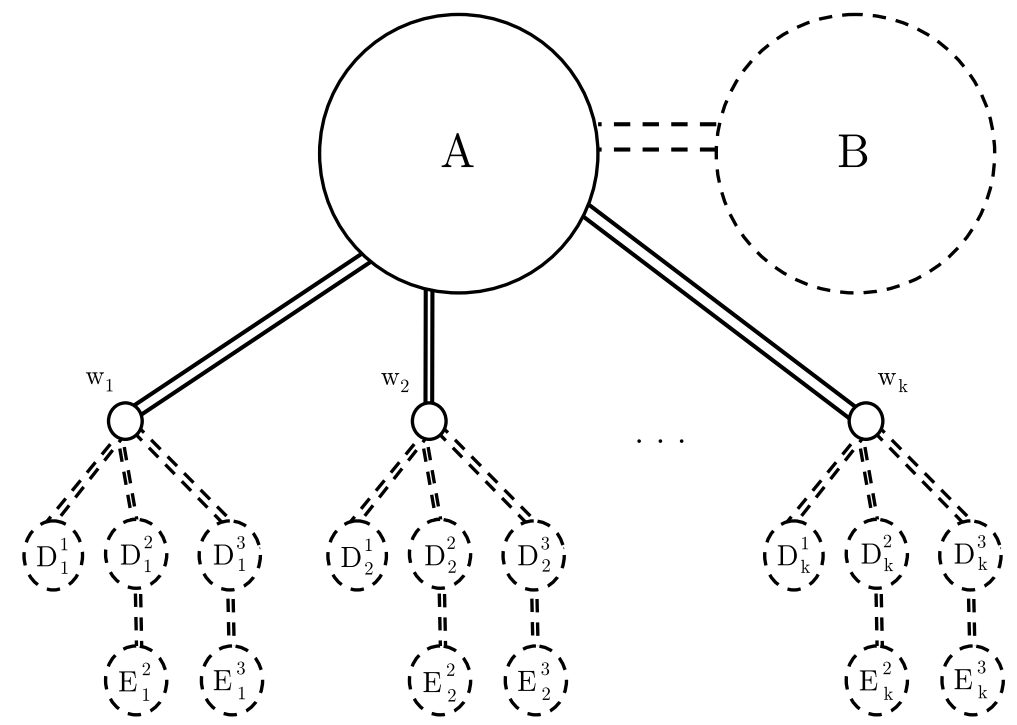

Figure 2: The graphs $G$ (in full lines) and $G^{\prime}$ (in dashed and full lines) from the proof of Theorem 6 .

Define $\mu: V\left(G^{\prime}\right) \rightarrow \mathbb{N}$ as follows: $\mu\left(v_{i}\right)=\mu\left(v_{i}^{\prime}\right)=2 i$ for each $i$ in $[n]$; $\mu\left(w_{j}\right)=\ell_{j}^{3}$ for each $j$ in $[k] ; \mu(w)=\ell_{j}^{i}-1$ for each $w \in D_{j}^{i}$ and $\mu(w)=\ell_{j}^{i-1}$ for each $w \in E_{j}^{i}$, for each suitable pair $(i, j)$.

Let $f: V\left(G^{\prime}\right) \rightarrow \mathbb{N}$ be a solution to $\left(G^{\prime}, \mu\right)$. The sets $A, B$ satisfy the conditions of Lemma 1 , so $f(v) \in L(v)$ for each $v \in A$. Let $w_{j} \in C$. Observe that, by construction, the vertices in each set $E_{j}^{i}$ are forced to use all and only the colors in $\left[\ell_{j}^{i-1}\right]$. Therefore, the vertices in each set $D_{j}^{i}$ are forced to use all and only the colors in the interval $\left[\ell_{j}^{i-1}+1, \ldots, \ell_{j}^{i}-1\right]$. Then $f\left(w_{j}\right) \in\left\{\ell_{j}^{1}, \ell_{j}^{2}, \ell_{j}^{3}\right\}=L\left(w_{j}\right)$ must hold.

Conversely, let $f$ be a solution to $(G, L)$. Then it can be extended to vertices of $D \cup E$ by assigning, for each suitable pair $(i, j)$, colors from $\left[\ell_{j}^{i-1}+1, \ldots, \ell_{j}^{i}-1\right]$ to vertices of $D_{j}^{i}$, and colors in $\left[\ell_{j}^{i-1}\right]$ to vertices of $E_{j}^{i}$. Since $B$ is anticomplete to $C \cup D \cup E$, we can use Lemma 1 to argue that $f$ can be extend to vertices of $B$ as well.

Putting things together, it follows that there exists a feasible coloring for $\left(G^{\prime}, \mu\right)$ if and only if there exists a feasible solution to the 3-SAT instance. 
Note that the size of $G^{\prime}$ is a polynomial in the size of the 3-SAT instance. We therefore conclude that the $\mu$-coloring problem is NP-complete on clique trees of height 3. It is trivial to extend this result to clique trees of fixed height $p \geq 4$ : we omit the details.

We now move to the second statement in the theorem. We therefore define an instance $\left(G^{\prime \prime}, P\right)$ of the precoloring extension problem such that the list-coloring instance $(G, L)$ has a solution if and only if $\left(G^{\prime \prime}, P\right)$ has a solution, where $G^{\prime \prime}$ is a clique tree of height 2 .

Let $A, C$ be as in the definition of $G, B=\left\{v_{1}^{\prime}, \ldots, v_{n}^{\prime}\right\}$ be a stable set disjoint from $A \cup C$. Let $E_{1}, \ldots, E_{n}$ be pairwise disjoint cliques, disjoint from $A \cup B \cup C$, such that for each $i \in[n],\left|E_{i}\right|=2 n-2$ and its vertices are precolored with (different) colors in $[2 n] \backslash\{2 i-1,2 i\}$. Let $E=\bigcup E_{i}$. Let $D_{1}, \ldots, D_{k}$ be pairwise disjoint cliques, disjoint from $A \cup B \cup C \cup E$, such that for each $i \in[k],\left|D_{i}\right|=2 n-3$ and its vertices are precolored with (different) colors in $[2 n] \backslash L\left(w_{i}\right)$. Let $D=\bigcup D_{i}$. $G^{\prime \prime}$ is then defined as follows: $V\left(G^{\prime \prime}\right)=A \cup B \cup C \cup D \cup E ; G^{\prime \prime}[A \cup C]=G, B$ is complete to $A, D_{i}$ is complete to $\left\{w_{j}\right\}$ for each $j \in[k], E_{i}$ is complete to $\left\{v_{i}^{\prime}\right\}$ for each $i \in[n]$, and there are no more edges in $G^{\prime \prime}$. Observe that $G^{\prime \prime}$ is a clique tree of height at most 2.

With similar arguments as those used above, it can be shown that $(G, L)$ has a solution if and only if $\left(G^{\prime \prime}, P\right)$ has a solution. Moreover, the size of $G^{\prime \prime}$ is a polynomial in the size of the 3-SAT instance. Therefore, the precoloring extension problem is NP-complete on clique trees of height 2. In fact, it is trivial to extend this result to clique trees of fixed height $p \geq 3$ : we omit the details.

Since the $(\gamma, \mu)$-coloring problem is a generalization of the precoloring extension problem, we have the following corollary.

Corollary 7. For each integer $p \geq 2$, the $(\gamma, \mu)$-coloring problem is NPcomplete on clique trees of height $p$.

\section{Unit interval graphs}

A graph $G$ is an interval graph if it is the intersection graph of a set of intervals over the real line. A unit interval graph (uig) is the intersection graph of a set of intervals of length one, while a proper interval graph is the intersection graph of a set of intervals where no interval is properly contained in another. A claw is the complete bipartite graph $K_{1,3}$.

Theorem 8. [19] The classes of unit interval graphs, proper interval graphs, and claw-free interval graphs coincide. 
Let $v_{1}, \ldots, v_{n}$ be an ordering of the vertices of a graph $G$. The ordering is consistent if there is no triple $i<j<k$ such that $v_{k} v_{i} \in E(G)$ and $v_{k} v_{j} \notin$ $E(G)$. If, in addition, there is no triple $i<j<k$ such that $v_{i} v_{k} \in E(G)$ and $v_{i} v_{j} \notin E(G)$ (equivalently, the reverse ordering is also consistent), the ordering is called proper consistent.

Theorem 9. [17, 18] A graph if an interval graph if and only if its vertices admit a consistent order, while it is a unit interval graph if and only if its vertices admit a proper consistent order.

The main result of this section is the following.

Theorem 10. The $\mu$-coloring problem is NP-complete on unit interval graphs.

Our proof is based on a reduction from the following restriction of the satisfiability problem, that is known to be NP-complete [8]:

Restricted 3-SAT (R3-SAT)

Input: A $t$-variables Boolean formula $\phi$ in conjunctive normal form with $k$ clauses such that: each variable $x_{1}, x_{2}, \ldots, x_{t}$ does not appear twice in the same clause, while it appears in at least two and at most three different clauses; each literal $x_{1}, \bar{x}_{1}, x_{2}, \bar{x}_{2}, \ldots, x_{t}, \bar{x}_{t}$, appears at most twice, and each clause contains at least two and at most three literals.

Goal: Determine if there exists a truth assignment $\nu$ satisfying $\phi$.

Given an R3-SAT instance $(\phi)$, we denote by $\operatorname{size}_{\phi}$ the number of bits required for a binary encoding of $(\phi)$. The definition is quite standard, and we refer the reader to textbooks for details (see for example [16]). For sake of clarity, we start with reducing R3-SAT to a list-coloring problem with some parity constraints, which we call Parity list coloring ( $P L C$ in short). We will then show how to complete the proof, linking PLC to the $\mu$-coloring problem on uig.

PARITY LIST COLORING (PLC)

Input: A graph $G$, a finite list $L(v) \subseteq \mathbb{N}$ for each vertex $v \in V(G)$, and a partition $\mathcal{F}$ of $V(G)$ into classes.

Goal: Find a list-coloring of $G$ such that all the vertices in a same class are assigned colors with the same parity.

We say that a set $F \in \mathcal{F}$ is trivial if $|F|=1$, non-trivial otherwise. 


\subsection{From satisfiability to parity list coloring}

As we are showing, PLC is NP-complete on complete graphs (recall that list-coloring is easy on such graphs). We first show how to associate to some instance $(\phi)$ of R3-SAT an instance $(G, L, \mathcal{F})$ of PLC. We assume that we are given some ordering $c_{1}, \ldots, c_{k}$ on the clauses of $(\phi)$, and associate therefore to $(\phi)$ a string $\ell_{1} \ldots \ell_{y}$ of characters from the alphabet of literals. For instance, if $\phi=\left(x_{1} \vee \bar{x}_{2}\right) \wedge\left(x_{1} \vee x_{2} \vee \bar{x}_{3}\right) \wedge\left(\bar{x}_{1} \vee x_{3}\right)$, we associate to $\phi$ the string $\ell_{1} \ldots \ell_{y} \equiv x_{1} \bar{x}_{2} x_{1} x_{2} \bar{x}_{3} \bar{x}_{1} x_{3}$. It follows from the definition of R3-SAT that $y \leq 3 t$ and $y \leq 3 k$.

We consider a clause as a set of characters, and associate to each clause $c_{j}, j \in[k]$, the set $\alpha\left(c_{j}\right)=\left\{1 \leq i \leq y: \ell_{i} \in c_{j}\right\}$ and to each variable $x_{i}$, $i \in[t]$, the set $\beta\left(x_{i}\right)=\left\{1 \leq i \leq y: \ell_{i} \equiv x_{i}\right.$ or $\left.\ell_{i} \equiv \bar{x}_{i}\right\}$. If we refer again to the previous example, we have that $\alpha\left(c_{1}\right)=\{1,2\}, \alpha\left(c_{2}\right)=\{3,4,5\}$, $\alpha\left(c_{2}\right)=\{6,7\}, \beta\left(x_{1}\right)=\{1,3,6\}, \beta\left(x_{2}\right)=\{2,4\}$ and $\beta\left(x_{3}\right)=\{5,7\}$.

We exploit some ideas from Jansen [12] and associate to $(\phi)$ an instance $\left(G_{\phi}, L_{\phi}, \mathcal{F}_{\phi}\right)$ of parity list coloring, that is defined as follows. $G_{\phi}$ is the complete graph of size $y+k$. The vertices of $G_{\phi}$ are partitioned into two sets: $T=\left\{v_{1}, \ldots, v_{y}\right\}$ and $U=\left\{v_{y+1}, v_{y+2}, \ldots, v_{y+k}\right\}$. The lists $L_{\phi}$ of feasible colors of $V\left(G_{\phi}\right)$ are defined as follows:

for $p \in[y], L_{\phi}\left(v_{p}\right)=\{2 p, 2 p+1\}$;

for $j \in[k], L_{\phi}\left(v_{y+j}\right)=\left\{2 h: h \in \alpha\left(c_{j}\right)\right.$ and $\ell_{h} \equiv x_{i}$, for some $\left.i \in[t]\right\} \cup$ $\left\{2 h+1: h \in \alpha\left(c_{j}\right)\right.$ and $\ell_{h} \equiv \bar{x}_{i}$, for some $\left.i \in[t]\right\}$.

Finally, let $\mathcal{F}_{\phi}=\left\{\left\{v_{i}, i \in \beta\left(x_{1}\right)\right\}, \ldots,\left\{v_{i}, i \in \beta\left(x_{t}\right)\right\},\left\{v_{y+1}\right\}, \ldots,\left\{v_{y+k}\right\}\right\}$.

Lemma 11. There exists a feasible list-coloring for $\left(G_{\phi}, L_{\phi}, \mathcal{F}_{\phi}\right)$ if and only if there exists a feasible truth assignment for $(\phi)$, and one can construct one from the other in a time polynomial in size ${ }_{\phi}$.

Proof. The "if" part: let $\nu$ be a feasible assignment for $(\phi)$. Consider a coloring $f$ defined as follows: for $p \in[y], f\left(v_{p}\right)=2 p+1$ if the variable corresponding to $\ell_{p}$ is true in $\nu$, it is $f\left(v_{p}\right)=2 p$ otherwise; for $j \in[k]$, we choose a literal $\ell_{m}$ of $c_{j}$ that is true (there is at least one since $\nu$ is a feasible truth assignment), and set $f\left(v_{y+j}\right)=2 m+1$ if $\ell_{m}$ corresponds to a negated variable, $f\left(v_{y+j}\right)=2 m$ otherwise. It is straightforward to check that $f$ is feasible for $\left(G_{\phi}, L_{\phi}, \mathcal{F}_{\phi}\right)$.

The "only if" part: from a feasible list-coloring, we define a truth assignment $\nu$ as follows: a variable $\nu\left(x_{i}\right)$ is true if each vertex $v_{h}$ with $h \in \beta\left(x_{i}\right)$, has odd color, it is false otherwise. It is straightforward to check that $\nu$ is a 
feasible truth assignment. We conclude by pointing out that both producing a feasible truth assignment from a feasible coloring, and the converse operation can be performed in time polynomial in $\operatorname{size}_{\phi}$.

As an immediate corollary of the previous lemma, we obtain that PLC is NP-complete on cliques.

\subsection{From satisfiability to $\mu$-coloring on unit interval graphs}

In order to show that $\mu$-coloring is NP-complete on uigs, we associate with each instance $(\phi)$ of R3-SAT an instance $(G, \mu)$, with $G$ a uig. We postpone the complete definition of $(G, \mu)$, that is rather technical, to Section 3.3, but give here some crucial properties. Namely, $(G, \mu)$ is such that:

(P1) $V(G)=U \cup U^{\prime} \cup T \cup T^{\prime} \cup\left\{v^{\star}\right\}$.

(P2) $G[U \cup T]$ is isomorphic to $G_{\phi}$.

(P3) $G\left[T \cup T^{\prime} \cup\left\{v^{\star}\right\}\right]$ (resp. $G\left[U \cup U^{\prime}\right]$ ) is a uig admitting a proper consistent ordering where the vertices of $T \cup\left\{v^{\star}\right\}$ (resp. $U$ ) are last (resp. first).

(P4) $N\left(v^{\star}\right)=U \cup T ; U^{\prime}\left(\right.$ resp. $\left.T^{\prime}\right)$ is anti-complete to $T \cup T^{\prime}$ (resp. $U \cup U^{\prime}$ ).

(P5) For each $v \in U \cup T, \mu(v)=\max _{h \in L_{\phi}(v)} h ; \mu\left(v^{\star}\right)=1$.

(P6) $(G, \mu)$ can be built in time polynomial in size ${ }_{\phi}$.

Here $\left(G_{\phi}, L_{\phi}, \mathcal{F}_{\phi}\right)$ is the instance of PLC that is defined from $(\phi)$, as in Section 3.1.

Lemma 12. $G$ is a uig.

Proof. Observe that $U$ is complete to $T \cup\left\{v^{\star}\right\}$ and there are no more edges from $U \cup U^{\prime}$ to $T \cup T^{\prime} \cup\left\{v^{\star}\right\}$. The statement then follows from (P3).

It follows from Lemma 11 that, in order to prove Theorem 10, it is enough to prove the next lemma. Note that, with a slight abuse, we often look at $G_{\phi}$ as the subgraph of $G$ induced by $U \cup T$. Moreover, if $f$ is a coloring for a graph $G$ and $X \subseteq V(G)$, we denote by $f[X]$ the restriction of $f$ to $X$.

Lemma 13. If $f$ is a $\mu$-coloring for $(G, \mu)$, then $f\left[V\left(G_{\phi}\right)\right]$ is a feasible listcoloring for $\left(G_{\phi}, L_{\phi}, \mathcal{F}_{\phi}\right)$; vice versa every feasible coloring for $\left(G_{\phi}, L_{\phi}, \mathcal{F}_{\phi}\right)$ can be extended in time polynomial in size $\phi_{\phi}$ to a feasible coloring for $(G, \mu)$. 
We divide the proof of Lemma 13 into the proof of two lemmas, whose statements need a few definitions.

Our first aim is to define a "restriction" of $\left(G_{\phi}, L_{\phi}, \mathcal{F}_{\phi}\right)$ to $G[U]$ and $G[T]$. First, we let $L_{\phi}[U]$ (resp. $L_{\phi}[T]$ ) be the restriction of $L_{\phi}$ to $U$ (resp. $T)$. We also consider the restriction of $\mathcal{F}_{\phi}$ to $T$ and $U$ : this deserves a few words. First note that the definition of $\mathcal{F}_{\phi}$ is such that it does indeed induce a partition of $U$ and a partition of $T$. Moreover, the partition of $U$ is only composed of trivial sets, thus the parity constraints associated to vertices of $U$ can be neglected. Thus, $\mathcal{F}_{\phi}$ is essentially a partition of $T$ and, in the following, with a slight abuse, we will also refer to $\mathcal{F}_{\phi}$ as a partition of $T$. Therefore, the restriction of $\left(G_{\phi}, L_{\phi}, \mathcal{F}_{\phi}\right)$ to $G[U]$ is simply an instance $\left(G[U], L_{\phi}[U]\right)$ of list-coloring, while the restriction of $\left(G_{\phi}, L_{\phi}, \mathcal{F}_{\phi}\right)$ to $G[T]$ is an instance $\left(G[T], L_{\phi}[T], \mathcal{F}_{\phi}\right)$ of PLC.

Finally, we associate to an instance $(G, \mu)$ of the $\mu$-coloring on $G$ two "sub-instances" $\left(G\left[U \cup U^{\prime}\right], \mu\right)$ and $\left(G\left[T \cup T^{\prime} \cup\left\{v^{\star}\right\}\right], \mu\right)$, where we are slightly abusing notations since we are identifying $\mu$ respectively with its restriction to $U \cup U^{\prime}$ and to $T \cup T^{\prime} \cup\left\{v^{\star}\right\}$.

Lemma 14. If $f$ is a $\mu$-coloring for $\left(G\left[U \cup U^{\prime}\right], \mu\right)$, then $f[U]$ is a listcoloring for $\left(G_{\phi}[U], L_{\phi}[U]\right)$; vice versa every list-coloring for $\left(G_{\phi}[U], L_{\phi}[U]\right)$ can be extended in time polynomial in size $\phi_{\phi}$ to a $\mu$-coloring for $\left(G\left[U \cup U^{\prime}\right], \mu\right)$.

Lemma 15. If $f$ is a $\mu$-coloring for $\left(G\left[T \cup T^{\prime} \cup\left\{v^{\star}\right\}\right], \mu\right)$, then $f[T]$ is a feasible list-coloring for $\left(G_{\phi}[T], L_{\phi}[T], \mathcal{F}_{\phi}\right)$; vice versa every feasible listcoloring for $\left(G_{\phi}[T], L_{\phi}[T], \mathcal{F}_{\phi}\right)$ can be extended in time polynomial in size to a $\mu$-coloring for $\left(G\left[T \cup T^{\prime} \cup\left\{v^{\star}\right\}\right], \mu\right)$.

Assume now that Lemma 14 and 15 hold. As we show in the following, then Lemma 13 holds too.

In order to prove the first statement of Lemma 13, we consider a $\mu$ coloring $f$ for $(G, \mu)$. The restrictions $f\left[U \cup U^{\prime}\right]$ and $f\left[T \cup T^{\prime} \cup\left\{v^{\star}\right\}\right]$ are trivially a $\mu$-coloring for $\left(G\left[U \cup U^{\prime}\right], \mu\right)$ and a $\mu$-coloring for $\left(G\left[T \cup T^{\prime} \cup\left\{v^{\star}\right\}\right], \mu\right)$, respectively. It follows from Lemma 14 and 15 that $f[U]$ is a list-coloring for $\left(G_{\phi}[U], L_{\phi}[U]\right)$ and $f[T]$ is a feasible list-coloring for $\left(G_{\phi}[T], L_{\phi}[T], \mathcal{F}_{\phi}\right)$. We claim that $f[U \cup T]$ determines a feasible list-coloring for $\left(G_{\phi}, L_{\phi}, \mathcal{F}_{\phi}\right)$. That is easy to check, as soon as we observe that, for each vertex $u \in U$ and $t \in T, f(u) \neq f(t)$, since $T \cup U$ is a clique of $G$.

We now show the second statement of Lemma 13 . Consider therefore a feasible coloring for $\left(G_{\phi}, L_{\phi}, \mathcal{F}_{\phi}\right)$. Trivially, $f[U]$ is a coloring for $\left(G_{\phi}[U], L_{\phi}[U]\right)$ and $f[T]$ is a feasible coloring for $\left(G_{\phi}[T], L_{\phi}[T], \mathcal{F}_{\phi}\right)$. It follows from Lemma 14 and 15 that $f[U]$ can be extended to a $\mu$-coloring 
$f^{\prime}$ for $\left(G\left[U \cup U^{\prime}\right], \mu\right)$ and $f[T]$ can be extended to a $\mu$-coloring $f^{\prime \prime}$ for $\left(G\left[T \cup T^{\prime} \cup\left\{v^{\star}\right\}\right], \mu\right)$. Observe also that, for each vertex $u \in U$ and $t \in T$, $f(u) \neq f(t)$, since $T \cup U$ is a clique of $G_{\phi}$. Moreover, $f^{\prime \prime}\left(v^{\star}\right)=1$ (since $\mu\left(v^{\star}\right)=1$ ) and $f(u) \neq 1$, for $u \in U$ (color 1 does not belong to $L_{\phi}(u)$ ). Then it is easy to check that the union of $f^{\prime}$ and $f^{\prime \prime}$ determines a $\mu$-coloring for $G$, and that $f, f^{\prime}$ can be obtained in time polynomial in size ${ }_{\phi}$.

In the rest of the paper, we therefore build the graph $G$ as to satisfy properties (P1) to (P6), and prove Lemma 14 and 15.

\subsection{Building up the uig $G$}

In this section we construct graph $G$, describing explicitly the graphs $G\left[U \cup U^{\prime}\right]$ and $G\left[T \cup T^{\prime} \cup\left\{v^{\star}\right\}\right]$ and a proper consistent ordering of their vertices where vertices of $U$ (resp. $T \cup\left\{v^{\star}\right\}$ ) are first (resp. last).

\subsubsection{The graph $G\left[U \cup U^{\prime}\right]$ and the proof of Lemma 14}

Let $D=\bigcup_{v \in U} L_{\phi}(v)$. Let $U^{\prime}=\left\{w_{1}, \ldots, w_{2 y+1}\right\}$. The edges of $G\left[U \cup U^{\prime}\right]$ are as follows: $U, U^{\prime}$ are cliques; vertices $w_{i}$ with $i \in[2 y+1] \backslash D$ are complete to $U$; for $i \in D$, a vertex $v$ of $U$ is adjacent to $w_{i}$ if and only if $i<\min _{h \in L_{\phi(v)}} h$.

The proposed order of the vertices starts by the vertices of $U, v_{y+1}, \ldots$, $v_{y+k}$, followed by the vertices $w_{i}$ in $U^{\prime}$ with $i \in[2 y+1] \backslash D$, and finally by the vertices $w_{i}$ in $U^{\prime}$ with $i \in D$, ordered by their index.

Note that in the list-coloring instance $\left(G_{\phi}[U], L_{\phi}\right)$, for $j \in[k-1]$, each color in $L_{\phi}\left(v_{y+j+1}\right)$ is strictly greater than all the colors in $L_{\phi}\left(v_{y+j}\right)$. So $N\left[v_{y+1}\right]=\left\{w_{i}: i \in[2 y+1] \backslash D\right\}$, and $N\left[v_{y+j+1}\right]=N\left[v_{y+j}\right] \cup\left\{w_{i}: i \in\right.$ $\left.L_{\phi}\left(v_{y+j}\right)\right\}$, for each $j \in[k-1]$. Taking this into account, it is not hard to see that the order proposed above is a proper consistent order, thus $G\left[U \cup U^{\prime}\right]$ is a uig. An example can be seen in Figure 3.

As we already mentioned, for each $v \in U, \mu(v)=\max _{h \in L_{\phi}(v)} h$. Finally, we define $\mu$ over $U^{\prime}$ as $\mu\left(w_{i}\right)=i$ for each $i \in[2 y+1]$.

Proof of Lemma 14. Consider a $\mu$-coloring $f$ of $G\left[U \cup U^{\prime}\right]$. First note that the vertices in $U^{\prime}$ form a clique, and for each $i \in[2 y+1], \mu\left(w_{i}\right)=i$; this implies that $f\left(w_{i}\right)=i$ for each $i \in[2 y+1]$. Now, let $v \in U$. Since $\mu(v) \leq 2 y+1, f(v)$ is a color used by some vertex in $U^{\prime}$ nonadjacent to $v$. By definition of $\left(G\left[U \cup U^{\prime}\right], \mu\right)$, a vertex $w_{i} \in U^{\prime}$ is nonadjacent to $v$ if and only if either $i>\mu(v)$ or $i \in L_{\phi}(v)$. Since $f$ is a $\mu$-coloring, it follows that $f(v) \in L_{\phi}(v)$. Thus, $f[U]$ is a feasible solution for $\left(G_{\phi}[U], L_{\phi}\right)$. Conversely, given a feasible list-coloring of $\left(G_{\phi}[U], L_{\phi}\right)$, we can extend it to a $\mu$-coloring of $\left(G\left[U \cup U^{\prime}\right], \mu\right)$, by giving to each vertex $w$ of $U^{\prime}$ color $\mu(w)$. 


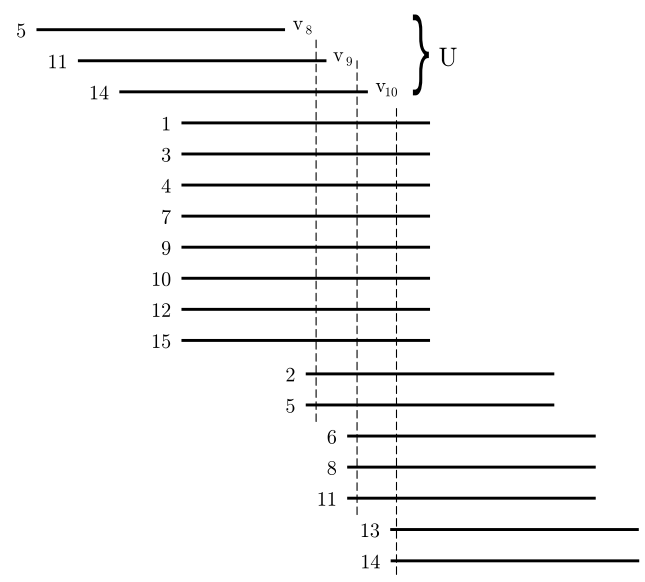

Figure 3: A unit interval representation of the instance $\left(G\left[U \cup U^{\prime}\right], \mu\right)$, for $\phi=\left(x_{1} \vee \bar{x}_{2}\right) \wedge$ $\left(x_{1} \vee x_{2} \vee \bar{x}_{3}\right) \wedge\left(\bar{x}_{1} \vee x_{3}\right)$ (the values of $\mu$ on each vertex are shown at the left of the corresponding interval). Recall that, for this instance, $y=7, k=3, U=\left\{v_{8}, v_{9}, v_{10}\right\}$, $L_{\phi}\left(v_{8}\right)=\{2,5\}, L_{\phi}\left(v_{9}\right)=\{6,8,11\}$ and $L_{\phi}\left(v_{10}\right)=\{13,14\}$.

\subsubsection{The graph $G\left[T \cup T^{\prime} \cup\left\{v^{\star}\right\}\right]$ and the proof of Lemma 15}

$G\left[T \cup T^{\prime} \cup\left\{v^{\star}\right\}\right]$ will be constructed through the following three intermediate steps: we start by building a gadget $H_{n}$, where $n$ is any positive integer. Then, for each $i \in[t]$, we build a graph $D^{i}$ starting from two copies of $H_{n}$. Last, we use graphs $D^{1}, \ldots, D^{t}$ to obtain $G\left[T \cup T^{\prime} \cup\left\{v^{\star}\right\}\right]$. We conclude the paragraph by showing that the latter graph satisfies Lemma 15 .

For each $n \in \mathbb{N}, n \geq 3, H_{n}$ is the graph on $4 n+8$ vertices, $V\left(H_{n}\right)=$ $\left\{z_{1}, \ldots, z_{4 n+8}\right\}$ defined below. Define the following disjoint subsets of $V\left(H_{n}\right)$ : $A_{1}^{0}=\left\{z_{2}, z_{3}\right\}, A_{2}^{0}=\left\{z_{4}, \ldots, z_{n+1}\right\}, B_{1}^{0}=\left\{z_{n+2}, z_{n+3}\right\}, B_{2}^{0}=\left\{z_{n+4}\right.$, $\left.\ldots, z_{2 n+1}\right\}, B_{2}^{1}=\left\{z_{2 n+8}, \ldots, z_{3 n+5}\right\}, B_{1}^{1}=\left\{z_{3 n+6}, z_{3 n+7}\right\}, A_{2}^{1}=\left\{z_{3 n+8}\right.$, $\left.\ldots, z_{4 n+5}\right\}, A_{1}^{1}=\left\{z_{4 n+6}, z_{4 n+7}\right\}$. The edges of $H_{n}$ are all and only the following: $\left\{z_{1}, \ldots, z_{2 n+1}\right\},\left\{z_{2 n+2}, \ldots, z_{2 n+7}\right\},\left\{z_{2 n+8}, \ldots, z_{4 n+8}\right\}$ are complete sets in $H_{n} ;\left\{z_{2 n+2}, z_{2 n+3}, z_{2 n+4}\right\}$ is complete to $A_{2}^{0} \cup B_{1}^{0} \cup B_{2}^{0} \cup B_{2}^{1}$; $\left\{z_{2 n+5}, z_{2 n+6}, z_{2 n+7}\right\}$ is complete to $B_{2}^{0} \cup B_{2}^{1} \cup B_{1}^{1} \cup A_{2}^{1}$; $B_{2}^{0}$ is complete to $B_{2}^{1}$. It is easy to see that the order $z_{1}, \ldots, z_{n}$ is a proper consistent order for $V\left(H_{n}\right)$ (see Figure 4 for a unit interval representation of $H_{n}$ ).

Remark 16. Graph $H_{n}$ is a symmetric proper interval graph, that is, the one-to-one correspondence $z_{j} \mapsto z_{4 n+9-j}$ is an automorphism of $H_{n}$.

Let now $n_{1}, n_{2} \in \mathbb{N}, n_{1}<n_{2} \leq n / 2$. Define the integers $a=2 n_{1}$ and 


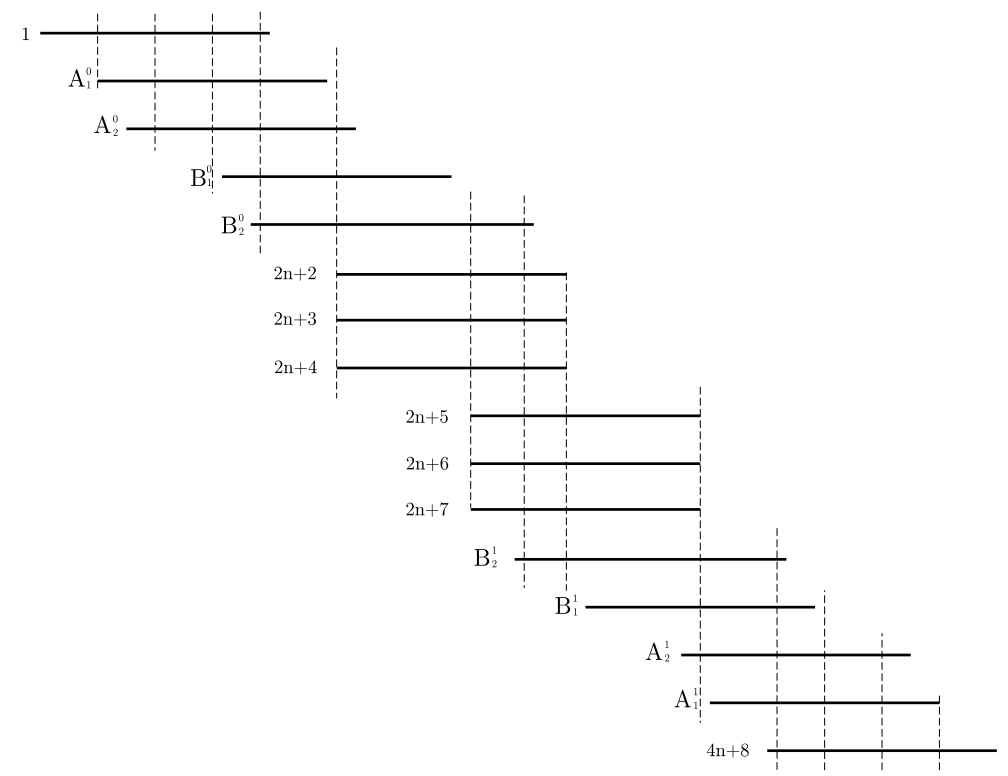

Figure 4: A unit interval representation of the graph $H_{n}$.

$b=2 n_{2}$ and consider the following list-coloring constraints $L_{a, b}$ :

- $L_{a, b}\left(z_{1}\right)=1$

- $L_{a, b}\left(z_{2}\right)=L_{a, b}\left(z_{n+2}\right)=\{a, a+1\}$;

- $L_{a, b}\left(z_{3}\right)=L_{a, b}\left(z_{n+3}\right)=\{b, b+1\}$;

- for $z_{j} \in A_{2}^{0}$,

$$
L_{a, b}\left(z_{j}\right)=L_{a, b}\left(z_{j+n}\right)= \begin{cases}\{2(j-3), 2(j-3)+1\} & \text { if } j<n_{1}+3 \\ \{2(j-2), 2(j-2)+1\} & \text { if } n_{1}+3 \leq j<n_{2}+2 \\ \{2(j-1), 2 j-1\} & \text { if } n_{2}+2 \leq j\end{cases}
$$

- $L_{a, b}\left(z_{2 n+2}\right)=\{a+1, b\}$;

- $L_{a, b}\left(z_{2 n+3}\right)=\{b+1,2 n+2\}$;

- $L_{a, b}\left(z_{2 n+4}\right)=\{1, a\}$;

- for $j \geq 2 n+5, L_{a, b}\left(z_{j}\right)=L_{a, b}\left(z_{4 n+9-j}\right)$.

Remark 17. Let $n_{1}, n_{2} \in \mathbb{N}, n_{1}<n_{2} \leq n / 2$, and $\left(H_{n}, L_{a, b}\right)$ defined as above. Then for each $j \in[n] \backslash\left\{n_{1}, n_{2}\right\}$, there exists a unique vertex $v \in A_{2}^{0}$ 
(resp. $\left.B_{2}^{0}, A_{2}^{1}, B_{2}^{1}\right)$ such that $L_{a, b}(v)=\{2 j, 2 j+1\}$, and conversely for each vertex $v \in A_{2}^{0}$ (resp. $\left.B_{2}^{0}, A_{2}^{1}, B_{2}^{1}\right)$ there exist a value $j \in[n] \backslash\left\{n_{1}, n_{2}\right\}$ such that $L_{a, b}(v)=\{2 j, 2 j+1\}$.

Lemma 18. For $a, b$ defined as above and $\mu: V\left(H_{n}\right) \rightarrow \mathbb{N}$ defined as $\mu(v)=$ $\max _{h \in L_{a, b}(v)} h$, the following properties hold:

(i) every solution of $\left(H_{n}, \mu\right)$ is a solution of $\left(H_{n}, L_{a, b}\right)$, and conversely every solution of $\left(H_{n}, L_{a, b}\right)$ is a solution of $\left(H_{n}, \mu\right)$.

(ii) in no feasible coloring $f$ of $\left(H_{n}, L_{a, b}\right)$, there exist $w, w^{\prime} \in A^{0}:=A_{1}^{0} \cup$ $A_{2}^{0}$ (resp. $\quad A^{1}:=A_{1}^{1} \cup A_{2}^{1}$ ) such that $f(w)=a, f\left(w^{\prime}\right)=b+1$, or $f(w)=a+1, f\left(w^{\prime}\right)=b$.

(iii) no pair of vertices of $A^{0}$ and $A^{1}$ share a color in any feasible coloring of $\left(H_{n}, L_{a, b}\right)$;

(iv) any coloring of $A^{0}$ (resp. $A^{1}$ ) that does not violate constraints from $L_{a, b}$ and from point (ii) can be extended in time polynomial in $n$ to a list-coloring of $\left(H_{n}, L_{a, b}\right)$.

Proof.

(i) One direction is trivial, thus we only need to show that for each coloring $f$ that is feasible for $\left(H_{n}, \mu\right), f(v) \in L_{a, b}(v)$ holds true for each $v \in V\left(H_{n}\right)$. Let $f$ be a feasible coloring of $\left(H_{n}, \mu\right)$. Since $\mu\left(z_{1}\right)=1, f\left(z_{1}\right)=1$. Recall that $A_{1}^{0} \cup A_{2}^{0} \cup B_{1}^{0} \cup B_{2}^{0}$ is a clique, and for each $i \in[n]$ there are exactly two vertices of this clique with $\mu=2 i+1$ (see Remark 17): as an immediate corollary of Lemma 1 (having excluded color 1 , that none of vertices from $A_{1}^{0} \cup A_{2}^{0} \cup B_{1}^{0} \cup B_{2}^{0}$ will be colored with, being they adjacent to $\left.z_{1}\right), f(w) \in L_{a, b}(w)$ for $w \in A^{0} \cup B_{1}^{0} \cup B_{2}^{0}$. Being $H_{n}$ symmetric (cfr. Remark 17), the same holds for $w \in A^{1} \cup B_{1}^{1} \cup B_{2}^{1}$. Vertex $z_{2 n+4}$ is adjacent to the clique $A_{2}^{0} \cup B_{2}^{0}$ which has $a-2$ vertices that can be colored with colors from 2 to $a-1$, so $f\left(z_{2 n+4}\right) \in\{1, a\}=L_{a, b}\left(z_{2 n+4}\right)$. Again by symmetry we conclude that $f\left(z_{2 n+5}\right) \in L_{a, b}\left(z_{2 n+5}\right)$. Vertex $z_{2 n+2}$ is adjacent to the clique $A_{2}^{0} \cup B_{2}^{0}$ that has $a-2$ vertices colored with colors from 2 to $a-1$ and $b-a-2$ colors between $a+2$ and $b-1$, so it can be colored only with $1, a, a+1$ and $b$. Furthermore it is adjacent to $z_{2 n+4}$ and $z_{2 n+5}$, so it cannot be colored with 1 and $a$ as well, thus the only colors left are those from its list. Again a symmetric argument works for $z_{2 n+7}$. In order to conclude the proof, one can easily show $f(w) \in L_{a, b}(w)$ for $w=z_{2 n+3}, z_{2 n+6}$ by using similar arguments as those used above. 
(ii) From Remark 17, $z_{2}$ and $z_{3}$ are the only vertices from $A^{0}$ that can be colored with $a, a+1, b, b+1$. In particular $L_{a, b}\left(z_{2}\right)=\{a, a+1\}$ and $L_{a, b}\left(z_{3}\right)=\{b, b+1\}$. Suppose first $f\left(z_{2}\right)=a$ and $f\left(z_{3}\right)=b+1$; then, $f\left(z_{n+2}\right)=a+1$ and $f\left(z_{n+3}\right)=b$, which implies that vertex $z_{2 n+2}$ cannot be colored. Now suppose $f\left(z_{2}\right)=a+1$ and $f\left(z_{3}\right)=b$, then the following colors are implied: $f\left(z_{n+2}\right)=a, f\left(z_{n+3}\right)=b+1$, $f\left(z_{2 n+3}\right)=2 n+2, f\left(z_{2 n+6}\right)=b+1, f\left(z_{2 n+4}\right)=1$, and $f\left(z_{2 n+5}\right)=a$. Since $L_{a, b}\left(z_{2 n+7}\right)=\{a+1, b\}$, either the pair of colors $a, a+1$ or $b, b+1$ are used by vertices adjacent to $z_{3 n+6}$ and $z_{3 n+7}$, which then cannot be colored. The symmetric argument works for $A^{1}$.

(iii) Let $f$ be a feasible coloring for $\left(H_{n}, L_{a, b}\right)$. Recall that $A^{0}=A_{1}^{0} \cup A_{2}^{0}$; pick any color $c$ such that $f(w)=c$ for some $c \in A_{2}^{0}$, and recall that $c \neq a, a+1, b, b+1$. Suppose first $c$ is even. By Remark 17, $c \neq a, a+1, b, b+1$ and there is a vertex $w^{\prime} \in B_{2}^{0}$ such that $L_{a, b}\left(w^{\prime}\right)=$ $\{c, c+1\}$, so $f\left(w^{\prime}\right)=c+1$. Repeating the same argument, $f\left(w^{\prime \prime}\right)=c$ for some $w^{\prime \prime} \in B_{2}^{1}$, and a $f\left(w^{\prime \prime \prime}\right)=c+1$ for some $w^{\prime \prime} \in A_{2}^{1}$. Since by Remark 17 no other vertex of $A^{1}$ can be colored with $c$, we conclude the proof for this case. Being the graph symmetric (cfr. Remark 16), we can reverse the argument and settle the case when $c$ is odd. Now pick any color $c$ such that $f(w)=c$ for some $w \in A_{1}^{0}=\left\{z_{2}, z_{3}\right\}$ and recall that $c \in\{a, a+1, b, b+1\}$. Being $f$ a feasible coloring for $\left(H_{n}, L_{a, b}\right)$, by part $(i i)$ of the Lemma, either $f\left(z_{2}\right)=a$ and $f\left(z_{3}\right)=b$, or $f\left(z_{2}\right)=a+1$ and $f\left(z_{3}\right)=b+1$. Suppose the first holds. Then, being $f$ feasible for $\left(H_{n}, L_{a, b}\right)$, the following colors are implied: $f\left(z_{n+2}\right)=$ $a+1, f\left(z_{n+3}\right)=b+1, f\left(z_{2 n+2}\right)=b, f\left(z_{2 n+3}\right)=2 n+2, f\left(z_{2 n+6}\right)=b+1$ and $f\left(z_{2 n+7}\right)=a+1$. Moreover, note now that $f\left(z_{2 n+5}\right)=1$, otherwise $z_{3 n+7}$ cannot be colored, so $f\left(z_{2 n+4}\right)=a$. Repeating the argument, we obtain that $f\left(z_{4 n+6}\right)=b+1$ and $f\left(z_{4 n+7}\right)=a+1$. Thus, we showed that if $f\left(z_{2}\right)=a$ and $f\left(z_{3}\right)=b$, and $f\left(z_{4 n+6}\right)=b+1 f\left(z_{4 n+7}\right)=a+1$. Being $z_{4 n+6}, z_{4 n+7}$ the only vertices from $A^{1}$ that can be colored with $a, a+1, b, b+1$, this concludes the proof for this case. We are left to settle the statement for the case $f\left(z_{2}\right)=a+1$ and $f\left(z_{3}\right)=b+1$ : note that this is implied by the previous one, since $H_{n}$ is symmetric.

(iv) Let us settle the case for $A^{0}$, following the other by symmetry. Repeating the arguments from the proof of part $(i i i)$, we observe that in any extension of a coloring $f$ of $A^{0}, B^{1}:=B_{1}^{1} \cup B_{2}^{1}$ must use the same colors of $A^{0}$, while $B^{0}:=B_{1}^{0} \cup B_{2}^{0}$ and $A^{1}$ colors $c+1$ for each color $c$ used by $A^{0}$ with $c$ even, and colors $c-1$ for each color $c$ used by $A^{0}$ 
with $c$ odd. Moreover, following again the proof of part (iii), vertices $z_{2 n+2}, \ldots, z_{2 n+7}$ can be assigned a color to make the coloring feasible.

For each $i \in[t]$, we now define a graph $D^{i}$ and an instance of PLC $\left(D^{i}, L^{i}, \mathcal{F}^{i}\right)$. For a given instance $\phi$ of R3-SAT and a fixed $i \in[t]$, let $H^{\prime}, H^{\prime \prime}$ be two copies of $H_{y}$, with $V\left(H^{\prime}\right)=\left\{z_{1}^{\prime}, \ldots, z_{4 y+8}^{\prime}\right\}, V\left(H^{\prime \prime}\right)=$ $\left\{z_{1}^{\prime \prime}, \ldots, z_{4 y+8}^{\prime \prime}\right\} . D^{i}$ is the graph obtained by $H^{\prime} \cup H^{\prime \prime}$ by adding all edges between $A^{1} \cup\left\{z_{4 y+8}^{\prime}\right\}$ from $H^{\prime}$ and $A^{0} \cup\left\{z_{1}^{\prime}\right\}$ from $H^{\prime \prime}$, and then contracting vertex $z_{4 y+8}^{\prime}$ of $H^{\prime}$ with vertex $z_{1}^{\prime \prime}$ of $H^{\prime \prime}$. With this definition, $z_{1}^{\prime}, \ldots, z_{4 y+8}^{\prime}, z_{2}^{\prime \prime}, \ldots, z_{4 y+8}^{\prime \prime}$ is a proper consistent ordering of $V\left(D^{i}\right)$, and both $\left\{z_{1}^{\prime}, \ldots, z_{4 y+8}^{\prime}\right\}$ and $\left\{z_{4 y+8}^{\prime}, z_{2}^{\prime \prime}, \ldots, z_{4 y+8}^{\prime \prime}\right\}$ induce $H_{y}$ on $D^{i}$ (see Figure 5). Thus, $D^{i}$ is a uig. We call $u_{j}^{i}:=z_{j}^{\prime}$ for $1 \leq j \leq 4 y+8, u_{j}^{i}:=z_{j-(4 y+7)}^{\prime \prime}$ for $4 y+9 \leq j \leq 8 y+15, E^{i}=\left\{u_{2}^{i}, \ldots, u_{y+1}^{i}\right\}, F^{i}=\left\{u_{7 y+15}^{i}, \ldots, u_{8 y+14}^{i}\right\}$. Recall $E^{i}=A^{0}$ from $H^{\prime}$ and $F^{i}=A^{1}$ from $H^{\prime \prime}$.

Let $y_{i}$ be the number of occurrences of variable $x_{i}$ in $\phi$, so $y_{i} \in\{2,3\}$. Let $p_{i}^{1}<\ldots<p_{i}^{y_{i}}$ such that $\left\{p_{i}^{1}, \ldots, p_{i}^{y_{i}}\right\}=\beta\left(x_{i}\right)$. Define $L^{i}$ as $L_{2 p_{i}^{1}, 2 p_{i}^{2} \text { over }}$ $\left\{z_{1}^{\prime}, \ldots, z_{4 y+8}^{\prime}\right\}$ and $L_{2 p_{i}^{1}, 2 p_{i}^{y_{i}}}$ over $\left\{z_{4 y+8}^{\prime}, z_{2}^{\prime \prime}, \ldots, z_{4 y+8}^{\prime \prime}\right\}$ (it is well defined since $\left.L_{2 p_{i}^{1}, 2 p_{i}^{2}}\left(z_{4 y+8}^{\prime}\right)=L_{2 p_{i}^{1}, 2 p_{i}^{y_{i}}}\left(z_{1}^{\prime \prime}\right)=\{1\}\right)$. Set $\mu(u)=\max _{h \in L^{i}(u)} h$ for each $u \in V\left(D^{i}\right)$.

Last, let $\mathcal{F}^{i}$ be the partition of $V\left(D^{i}\right)$ whose only non-trivial set is $\{v \in$ $F^{i}: L^{i}(v)=\{2 p, 2 p+1\}$ and $\left.p \in \beta\left(x_{i}\right)\right\}$.

Lemma 19. For $D^{i}, L^{i}, \mathcal{F}^{i}, \mu$ defined as above, the following holds:

(1) $E^{i}$ and $F^{i}$ are cliques in $D^{i}, u_{1}^{i}$ is complete to $E^{i}$, while $u_{8 y+15}^{i}$ is complete to $F^{i}$.

(2) For each $i \in[t]$ and $p \in[y]$, there exists a unique vertex $v \in E^{i}$ (resp. $\left.F^{i}\right)$ such that $L^{i}(v)=\{2 p, 2 p+1\}$, and conversely for each vertex $v \in E^{i}$ (resp. $F^{i}$ ) there exist a value $p \in[y]$ such that $L^{i}(v)=\{2 p, 2 p+1\}$.

(3) $E^{i}$ and $F^{i}$ share no color in any feasible list-coloring of $\left(D^{i}, L^{i}\right)$.

(4) Every $\mu$-coloring of $D^{i}$ is a feasible coloring for $\left(D^{i}, L^{i}\right)$.

(5) Any feasible coloring of $\left(D^{i}, L^{i}\right)$ is a feasible coloring for $\left(D^{i}, L^{i}, \mathcal{F}^{i}\right)$.

(6) Any feasible solution to $\left(D^{i}\left[F^{i}\right], L^{i}, \mathcal{F}^{i}\right)$ can be extended in time polynomial in size ${ }_{\phi}$ to a feasible coloring for $\left(D^{i}, L^{i}\right)$. 


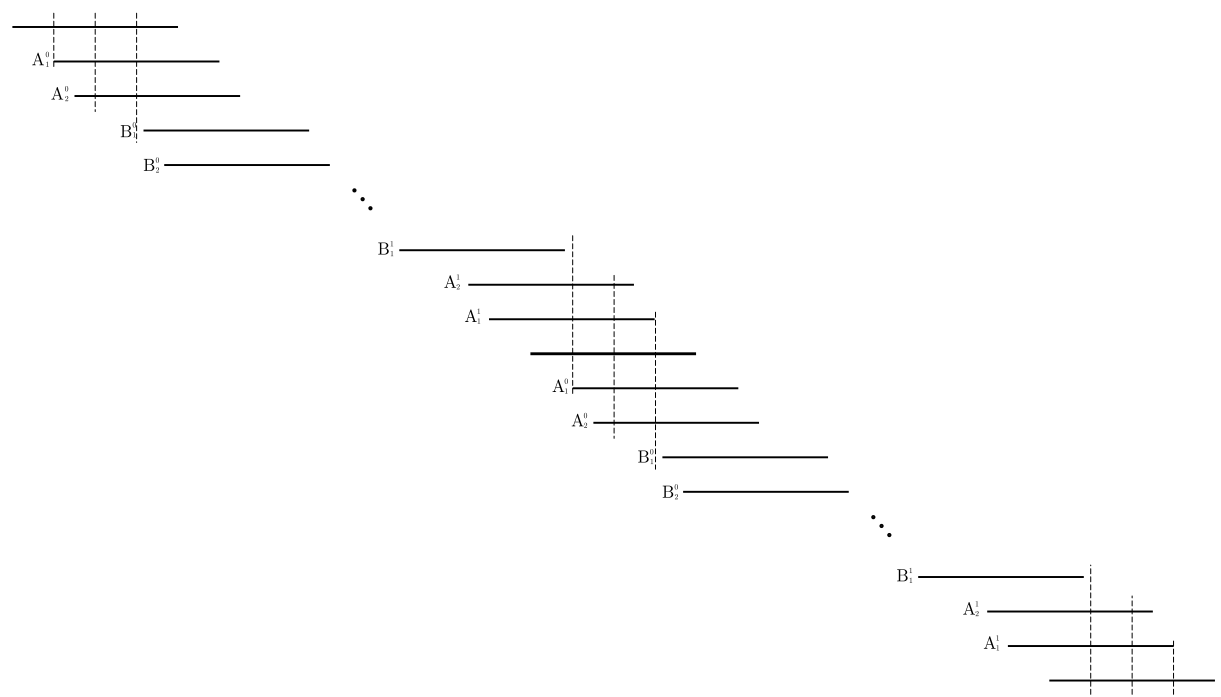

Figure 5: A unit interval representation of the graph $D^{i}$.

Proof. (1) and (2) are immediately shown true, by the very definition of $\left(D^{i}, L^{i}, \mathcal{F}^{i}\right)$ and Remark 17.

(3) From Lemma 18.iii applied to each copy of $H_{y}$, vertices of $E^{i}\left(A^{0}\right.$ of $\left.H^{\prime}\right)$ share no color with vertices of $A^{1}$ of $H^{\prime}$, and vertices of $F^{i}\left(A^{1}\right.$ of $H^{\prime \prime}$ ) share no color with vertices of $A^{0}$ of $H^{\prime \prime}$. Since $A^{1}$ of $H^{\prime}$ is complete to $A^{0}$ of $H^{\prime \prime}$, they also share no color. From Remark 17 applied to each copy of $H_{y}$, for each $j \in[y]$, there is exactly one vertex from $A^{0}$ and one from $A^{1}$ that can be colored with $\{2 j, 2 j+1\}$; being both sets cliques of size $y$, then a color is used in $A^{0}$ if and only if it is not used in $A^{1}$, and this concludes the proof.

(4) It follows from Lemma 18.i and the definition of $\left(D^{i}, L^{i}\right)$.

(5) Let $f$ be a feasible list-coloring of $\left(D^{i}, L^{i}\right)$. By the definition of the lists $L^{i}, F^{i}$ never uses simultaneously $2 p_{i}^{j}$ and $2 p_{i}^{j}+1$ in $f$, for $j \in\left[y_{i}\right]$. From Lemma 18.ii, $F^{i}$ uses either both $2 p_{i}^{1}$ and $2 p_{i}^{2}$ or both $2 p_{i}^{1}+1$ and $2 p_{i}^{2}+1$, while $E^{i}$ uses either both $2 p_{i}^{1}$ and $2 p_{i}^{y_{i}}$ or both $2 p_{i}^{1}+1$ and $2 p_{i}^{y_{i}}+1$, respectively. If $y_{i}=2$ we are done, so suppose $y_{i}=3$. From Remark 17, for $j=1,2,3$ there is exactly one vertex from $E^{i}$ and one from $F^{i}$ that can be colored with $\left\{2 p_{i}^{j}, 2 p_{i}^{j}+1\right\}$. Suppose first $F^{i}$ uses $2 p_{i}^{1}$; repeating the arguments used for $y_{i}=2$ and because of Claim (3), this can happen if and only if $F^{i}$ also uses $2 p_{i}^{2}$, and if and 
only if $F^{i}$ uses $2 p_{i}^{3}$. If $F^{i}$ uses $2 p_{i}^{1}+1$, the proof is symmetric. So $f$ is a feasible solution for $\left(D^{i}, L^{i}, \mathcal{F}^{i}\right)$.

(6) Consider now a feasible solution to $\left(D^{i}\left[F^{i}\right], L^{i}, \mathcal{F}^{i}\right)$. From Lemma 18.iv, we know that it can be extended to vertices of $H^{\prime \prime}$, since it satisfies conditions of Lemma 18.ii with respect to the list-coloring instance $\left(H^{\prime \prime}, L_{2 p_{i}^{1}, 2 p_{i}^{y_{i}}+1}\right)$, that are a subset of conditions imposed by $\mathcal{F}^{i}$. Repeating the argument used in the proof of Claim (3), we can extend that coloring to $A^{1}$ from $H^{\prime}$ by assigning in the only possible way the set of colors used by $F^{i}$ in $f$. This partial coloring of $H^{\prime}$ respects the constraints of Lemma 18.ii for $\left(H^{\prime}, L_{2 p_{i}^{1}, 2 p_{i}^{2}+1}\right)$, since they are a subset of the constraints imposed by $\mathcal{F}^{i}$ on the set of colors used by $F^{i}$. Thus, by Lemma 18.iv and since the only edges from $H^{\prime}$ to $H^{\prime \prime}$ are from $A^{1}$ to $A^{0}$, we can extend it to the rest of the graph $H^{\prime}$, obtaining a feasible list-coloring for $\left(D^{i}, L^{i}\right)$.

In order to define $G\left[T \cup T^{\prime} \cup\left\{v^{\star}\right\}\right]$, we first introduce a sequence of graphs $G^{1}, \ldots, G^{t}: G^{1}=D^{1}$; for $2 \leq i \leq t, G^{i}$ is the graph obtained from $G^{i-1} \cup D^{i}$ by adding all edges between $u_{7 y+15}^{i-1}, \ldots, u_{8 y+15}^{i-1}$ and $u_{1}^{i}, \ldots, u_{y+1}^{i}$, and then contracting $u_{8 y+15}^{i-1}$ with $u_{1}^{i}$. Last, define $G\left[T \cup T^{\prime} \cup\left\{v^{\star}\right\}\right]=G^{t}$.

It is immediate to check that for each $i \in[t], i \geq 2, G^{i}$ has $(8 y+14) i+1$ vertices, and $u_{1}^{1}, u_{2}^{1}, \ldots, u_{8 y+15}^{1}, u_{2}^{2}, u_{3}^{2}, \ldots, u_{8 y+15}^{t}$ is a proper consistent order of $V\left(G^{i}\right)$, thus $G^{i}$ is a uig. With a slight abuse of notation, for $i=1, \ldots, t$, we will say that $D^{i}$ is an induced subgraph of $G\left[T \cup T^{\prime} \cup\left\{v^{\star}\right\}\right]$, considering when necessary the vertex obtained by contracting $u_{1}^{i}$ (resp. $u_{8 y+15}^{i}$ ) as $u_{1}^{i}$ (resp. $u_{8 y+15}^{i}$ ) itself. Note that the definition of $\mu$ over $G\left[T \cup T^{\prime} \cup\left\{v^{\star}\right\}\right]$ is univocally determined by the definition of $\mu$ over the graphs $D^{i}$, since for $2 \leq i \leq t, \mu\left(u_{8 y+15}^{i-1}\right)=\mu\left(u_{1}^{i}\right)=1$.

Finally, we shall identify $T$ with the set $F^{t}$ and $v^{\star}$ with vertex $u_{8 y+15}^{t}$. Lemma 19.2 allows us to identify $T$ with $F^{t}$ in such a way that, for each $p \in[y]$, the vertex $v_{p}$ of $T$ corresponds to the only vertex $v$ in $F^{t}$ satisfying $L^{t}(v)=L_{\phi}\left(v_{p}\right)=\{2 p, 2 p+1\}$. Observe that $\mu\left(v^{\star}\right)=1$ and that, in the proper consistent order given, $T \cup\left\{v^{\star}\right\}$ are the last vertices.

Proof of Lemma 15. Let $f$ be a $\mu$-coloring for $\left(G\left[T \cup T^{\prime} \cup\left\{v^{\star}\right\}\right], \mu\right)$. In particular, for each $i \in[t], f\left[V\left(D^{i}\right)\right]$ is a $\mu$-coloring of $D^{i}$ and, by Lemma 19.4, it is also a feasible coloring for $\left(D^{i}, L^{i}\right)$. By Lemma $19.3, E^{i}$ and $F^{i}$ share no color in $f$, and since $\left|E^{i}\right|=\left|F^{i}\right|=y$ and no vertex in $E^{i} \cup F^{i}$ contains the color 1 in its list, each of them uses half of colors in the set $[2 y+1] \backslash\{1\}$. Be- 
sides, for each $i \in[t-1], F^{i} \cup E^{i+1}$ is a clique, so $F^{i}$ and $E^{i+1}$ share no color in $f$; iterating, we deduce that all the sets $F^{1}, \ldots, F^{t}$ use the same subset of colors. By Lemma 19.5, $f\left[V\left(D^{i}\right)\right]$ is a feasible solution for $\left(D^{i}, L^{i}, \mathcal{F}^{i}\right)$. This means that, in $f\left[F^{i}\right]$, the vertices whose lists are $\{2 p, 2 p+1\}$ with $p \in \beta\left(x_{i}\right)$ are assigned colors with the same parity. Since the sets $F^{1}, \ldots, F^{t}$ use the same subset of colors, all parity constraints in $\mathcal{F}_{\phi}$ are satisfied in $F^{t}$. Therefore $f[T]$ is a feasible list-coloring for $\left(G_{\phi}[T], L_{\phi}[T], \mathcal{F}_{\phi}\right)$.

Conversely, let $f$ be a feasible list-coloring for $\left(G_{\phi}[T], L_{\phi}[T], \mathcal{F}_{\phi}\right)$. Recall that we identify vertices of $T$ with vertices in $F^{t}$ in such a way that, for each $p \in[y]$, the vertex $v_{p}$ of $T$ corresponds to the only vertex $v$ in $F^{t}$ satisfying $L^{t}(v)=L_{\phi}\left(v_{p}\right)=\{2 p, 2 p+1\}$. So, $f\left[F_{t}\right]$ is a feasible solution to $\left(D^{t}\left[F^{t}\right], L^{t}, \mathcal{F}^{t}\right)$. By Lemma 19.6, it can be extended to a feasible list-coloring $f^{\prime}$ for $\left(D^{t}, L^{t}\right)$. By Lemma $19.3, E^{t}$ and $F^{t}$ share no color in $f^{\prime}$. Then we can extend $f^{\prime}$ to $F^{t-1}$ by assigning to it the set of colors $f\left(F^{t}\right)$. By Lemma 19.2, there is only one possible way such that each vertex $v$ receives a color in $L^{t-1}(v)$. Moreover, since $f$ is a feasible list-coloring for $\left(G_{\phi}[T], L_{\phi}[T], \mathcal{F}_{\phi}\right)$, then $f^{\prime}\left[F_{t-1}\right]$ is a feasible solution to $\left(D^{t-1}\left[F^{t-1}\right], L^{t-1}, \mathcal{F}^{t-1}\right)$. By applying iteratively Lemma 19.6 and these arguments and by the structure of $G\left[T \cup T^{\prime} \cup\left\{v^{\star}\right\}\right]$ and the definition of $\mu$, it follows that $f^{\prime}$ can be extended to a solution of $\left(G\left[T \cup T^{\prime} \cup\left\{v^{\star}\right\}\right], \mu\right)$.

In order to conclude the proof of Theorem 10, we are left to note that, by de definition of $(G, \mu)$, properties (P1)-(P6) clearly hold.

\section{References}

[1] C. Berge, Les problèmes de colorations en théorie des graphes, Publications de l'Institut de Statistique de l'Université de Paris 9 (1960), 123-160.

[2] M. Biro, M. Hujter, and Zs. Tuza, Precoloring extension. I. Interval graphs, Discrete Mathematics 100(1-3) (1992), 267-279.

[3] F. Bonomo and M. Cecowski, Between coloring and list-coloring: $\mu$ coloring, Electronic Notes in Discrete Mathematics 19 (2005), 117-123.

[4] F. Bonomo, G. Durán, and J. Marenco, Exploring the complexity boundary between coloring and list-coloring, Annals of Operations Research 169(1) (2009), 3-16.

[5] V. Cacchiani, A. Caprara, and P. Toth, Solving a real-world train unit assignment problem, ATMOS 2007 - 7th Workshop on Algorithmic 
Approaches for Transportation Modeling, Optimization, and Systems, Dagstuhl, Germany (C. Liebchen, R. K. Ahuja, and J. A. Mesa, eds.), 2007.

[6] F. Gavril, Algorithms for minimum coloring, maximum clique, minimum covering by cliques and maximum independent set of a chordal graph, SIAM Journal on Computing 1(2) (1972), 180-187.

[7] M. Grötschel, L. Lovász, and A. Schrijver, The ellipsoid method and its consequences in combinatorial optimization, Combinatorica 1 (1981), 169-197.

[8] D. Gusfield and R.W. Irving, The Stable Marriage Problem. Structure and algorithms, MIT Press, Cambridge, MA, 1989.

[9] P.L. Hammer and F. Maffray, Complete separable graphs, Discrete Applied Mathematics 27(1) (1990), 85-99.

[10] M. Hujter and Zs. Tuza, Precoloring extension. II. Graph classes related to bipartite graphs, Acta Mathematica Universitatis Comenianae 62(1) (1993), 1-11.

[11] M. Hujter and Zs. Tuza, Precoloring extension. III. Classes of perfect graphs, Combinatorics, Probability and Computing 5 (1996), 35-56.

[12] K. Jansen, Complexity results for the optimum cost chromatic partition problem, manuscript, 1997.

[13] K. Jansen and P. Scheffler, Generalized coloring for tree-like graphs, Discrete Applied Mathematics 75 (1997), 135-155.

[14] L. Lovász, Normal hypergraphs and the perfect graph conjecture, Discrete Mathematics 2(3) (1972), 253-267.

[15] D. Marx, Precoloring extension on unit interval graphs, Discrete Applied Mathematics 154(6) (2006), 995-1002.

[16] G. Nemhauser and L. Wolsey, Integer and Combinatorial Optimization, Wiley Interscience Series in Discrete Mathematics and Optimization, John Wiley \& Sons, New York, 1988.

[17] S. Olariu, An optimal greedy heuristic to color interval graphs, Information Processing Letters 37 (1991), 21-25. 
[18] J. Ramalingam and C. Pandu Rangan, A unified approach to domination problems on interval graphs, Information Processing Letters $\mathbf{2 7}$ (1988), 271-274.

[19] F.S. Roberts, Indifference graphs, In: Proof Techniques in Graph Theory (F. Harary, ed.), Academic Press, 1969, pp. 139-146.

[20] V. Vizing, Coloring the vertices of a graph in prescribed colors, Metody Diskretnogo Analiza 29 (1976), 3-10. 